\title{
Aspiración estereotáctica de una larva de Spirometra spp.
}

\author{
Joel Caballero, Iosmill Morales, Diana García, Idelmys Alarcón, Anay Torres y Gladys Sáez
}

\section{Stereotactic aspiration of Spirometra mansonides larvae}

Brain sparganosis is a non-common parasite infection by Diphyllobothrium or Spirometra mansonoides larvae. This last one is responsible for most of the infestations in humans. We report a 19 years male patient bearer of a brain sparganosis. The patient presented with headache and left hemiparesis. CT diagnosis of right thalamic lesions was made and aspiration biopsy was performed using stereotactic system, obtaining a whole and death larvae. Histopathology confirms a CNS parasitism and it was treated initially with albendazol. ELISA test confirmed Spirometra spp. infestation. The patient developed asymptomatic with total remission of the lesions. It constitutes the second report in Cuba of brain sparganosis.

Key words: Spirometra mansonides, brain sparganosis, stereotactic aspiration.

Palabras clave: Spirometra mansonides, esparganosis cerebral, aspiración estereotáctica.

\section{Introducción}

L a esparganosis humana es una infección parasitaria causada por las larvas plerocercoides de la familia Diphyllobotriae, género Spirometra. Este último es responsable de la mayoría de las infecciones en humanos. Se ha observado una distribución mundial con predominio en países asiáticos, especialmente China, Japón, Vietnam y Korea ${ }^{1,2}$; probablemente debido a hábitos alimentarios ${ }^{3}$. Aunque Sparganum compromete generalmente el tejido subcutáneo y/o muscular (esparganosis subcutánea), existen otras localizaciones no tan frecuentes como la esparganosis ocular, oral, visceral (pleural, de tracto urinario, escrotal, abdominal) y encefálica ${ }^{4,5}$. La localización encefálica es particularmente inusual ${ }^{1}$. El diagnóstico se basa en la clínica y exámenes de laboratorio, imagenológicos e histopatológicos ${ }^{3}$. La reacción tisular depende si el verme se encuentra viable o no. El compromiso cerebral se observa en forma de un granuloma o absceso ${ }^{6}$. Existe una sola comunicación previa en Cuba de una infestación cerebral por este parásito ${ }^{7}$.

\section{Caso clínico}

Varón de 19 años de edad, diestro, con antecedentes de ingerir con frecuencia carne mal cocida de serpiente. Consultó por una cefalea occipital opresiva, ligera, de dos semanas de evolución, asociada a una disminución gradual y progresiva de la fuerza muscular del hemicuerpo izquierdo y parestesias que comenzaron en la extremidad superior, hemicara y luego en el miembro inferior. $\mathrm{Al}$ examen físico se constató una hemiparesia izquierda proporcional y directa y una hemi-hipoestesia izquierda para todas las modalidades. Se planteó un síndrome piramidal y un síndrome talámico. Se realizó una tomografía axial computarizada (TAC) de encéfalo con contraste observándose cuatro lesiones de tálamo derecho con realce en anillo, centro hipodenso y concéntricas; la mayor de ellas con un diámetro antero-posterior de $18 \mathrm{~mm}$, transversal de $17 \mathrm{~mm}$ y altura de $18 \mathrm{~mm}$. Todas las lesiones estaban asociadas a edema perilesional moderado y con efecto de masa sobre el tercer ventrículo (Figura $1 \mathrm{~A} \mathrm{y} \mathrm{B).}$

Se indicaron glucocorticoides y realizó una biopsia guiada por estereotaxia con el paciente despierto. Previo anestesia del cuero cabelludo con lidocaína al 0,5\% se colocó el marco cubano Esteroflex ${ }^{\circledR}$. Se realizó la TAC de planificación, se calcularon las coordenadas cartesianas y se planificó el punto de entrada y las trayectorias mediante el programa Stassis versión 3.0. Se identificó como punto " $T$ " un área de calcificación perteneciente a las coordenadas $\mathrm{X}=85, \mathrm{Y}=89, \mathrm{Z}=36$, como punto "T2" una zona extremo lateral con realce perteneciente a las coordenadas $\mathrm{X}=80, \mathrm{Y}=91, \mathrm{Z}=36$ y un punto "T3" que correspondió a la lesión en anillo más inferior mediante las coordenadas $X=84, Y=93, Z=40$. Bajo anestesia local se realizó incisión y agujero de trépano sobre el punto de Koscher. Se realizó coagulación dural y apertura cruciforme, coagulación y apertura pial. Se procedió a tomar cuatro muestras en diferentes direcciones horarias $(6,12$, 18 y 24 horas) de cada uno de los blancos determinados previamente. Se observó en una de las muestras correspondientes a un nódulo realzado, la salida de un posible verme de coloración blanquecina e inmóvil. El paciente
Instituto Nacional de Oncología y Radiobiología. La Habana, Cuba.

Neurocirugía (JC, IM)

Hospital Gonzáles Coro, Cuba. Anestesiología (DG)

Centro de Referencia de Ateroesclerosis de La Habana. Cuba.

Anatomía patológica (IA, AT) Medicina Interna y Urgencias Médicas (GS)

Los autores declaran no tener conflicto de intereses. Fuente de financiamiento: Instituto Nacional de Oncología y Radiobiología.

Recibido: 3 de enero de 2015 Aceptado: 21 de mayo de 2015

Correspondencia a: Joel Caballero García joelcg@infomed.sld.cu 

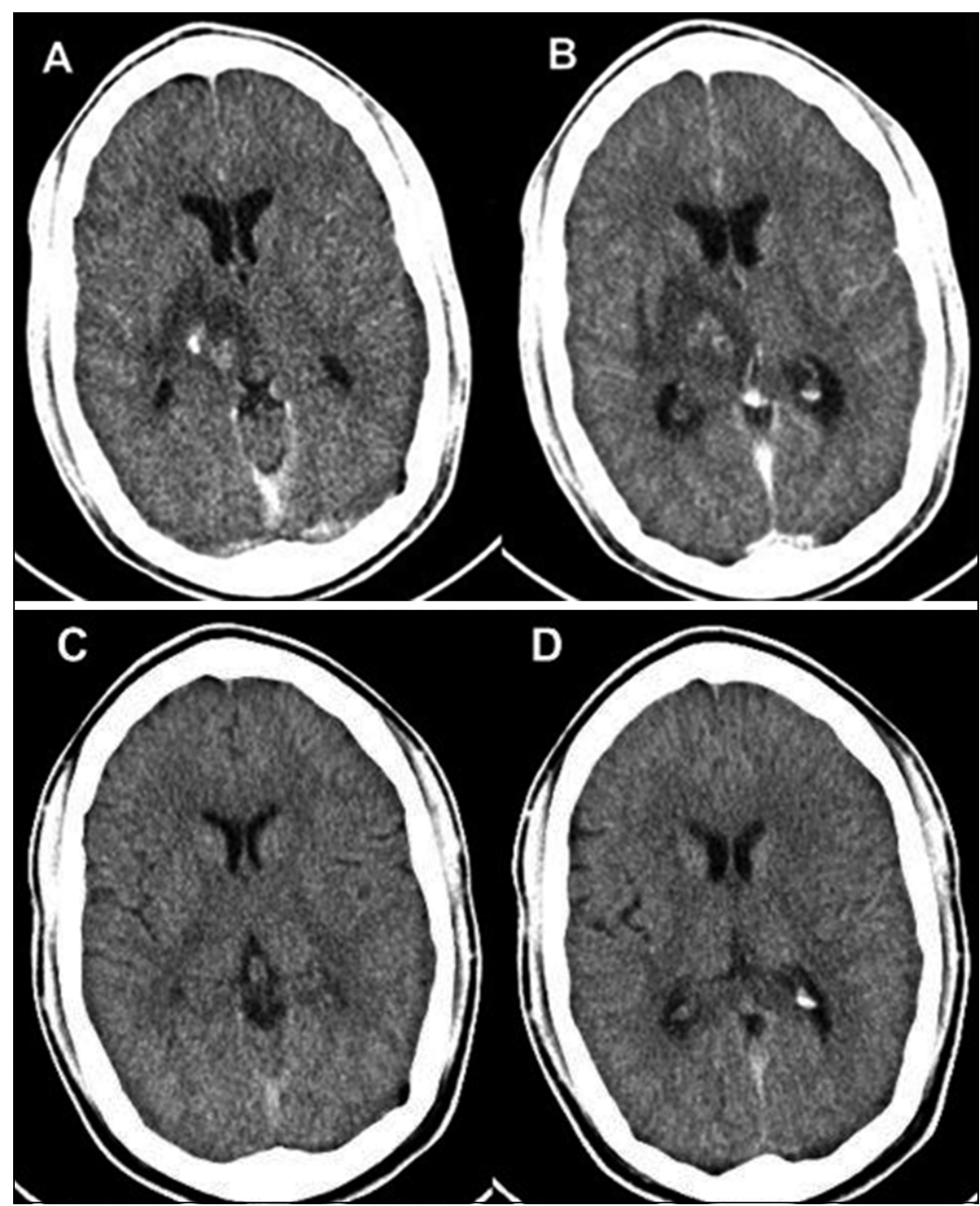

Figura 1 A y B. Tomografía computarizada preoperatoria. A: Se observan calcificaciones, nódulos y edema talámico derecho. B: Se observa el realce en anillo multilobulado. C y D: Tomografía computarizada de control a los dos meses de la cirugía. Se observa remisión total de las lesiones y del edema cerebral.

Figura 2. Durante la aspiración quirúrgica estereotáctica con el paciente despierto.

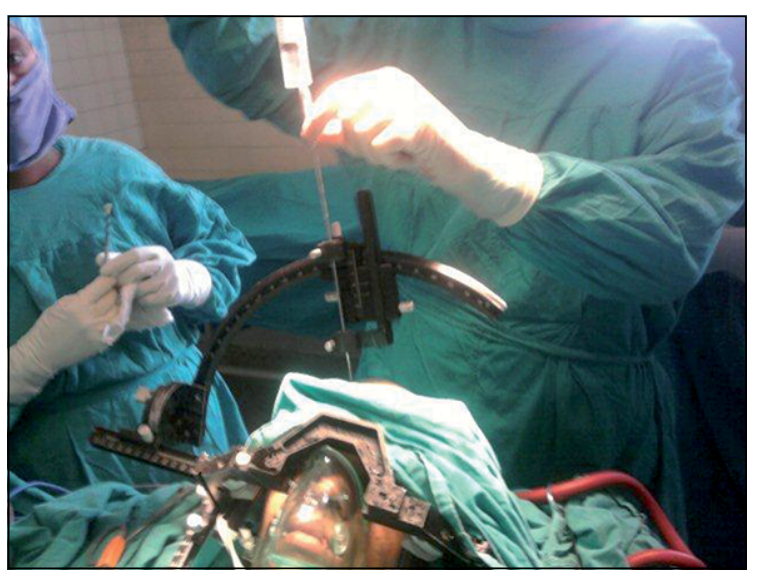

Figura 3. Imagen panorámica de un corte longitudinal de la larva
plerocercoide de Spirometra spp. Se observa la pseudosegmentación.

Figura 3. Imagen panorámica de un corte longitudinal de la larva
plerocercoide de Spirometra spp. Se observa la pseudosegmentación.

presentó un incremento transitorio de las parestesias durante el procedimiento quirúrgico sin otra complicación (Figura 2).

Se realizó una TAC post-quirúrgica inmediata en la que se observó escaso aire en el sitio de la biopsia sin signos de hemorragia u otra complicación y una TAC a los dos meses en la que se observó remisión total de las lesiones (Figura $1 \mathrm{C}$ y D). El estudio histopatológico mostró un segmento del parásito sólido, irregular, rodeado de hialinización y fibrosis, con variables calcificaciones y homogéneamente eosinofílico (Figura 3). No se encontró escólex. Inicialmente se indicó tratamiento con albendazol en esquema de $15 \mathrm{mg}$ al día, en tres dosis diarias. El test de ELISA en sangre y líquido cefalorraquídeo (LCR) inicial fue positivo para la inmunoglobulina Sparganumespecífica (IgG).

La evolución del paciente fue satisfactoria, a las ocho semanas desaparecieron todos los síntomas y se incorporó a su vida habitual. El test de ELISA en sangre y LCR de control a las 12 semanas fue negativo.

\section{Discusión}

La neurohelmintiasis en Cuba constituye una entidad infrecuente. Inicialmente se sospechó la presencia de una neurocisticercosis, aunque los escasos reportes se refieren a casos importados y el paciente no tenía antecedentes de viajes al exterior. Por otra parte, existe una comunicación previa de una aspiración estereotáctica de una larva viva de Spirometra spp. en el Centro Internacional de Restauración Neurológica (CIREN) ${ }^{7}$. La forma adulta de la Spirometra spp. vive en el intestino del perro y el gato. Las vías de infestación del humano ocurren por: ingestión de agua contaminada por copépodos infestados con procercoides de Spirometra; ingestión de hospederos

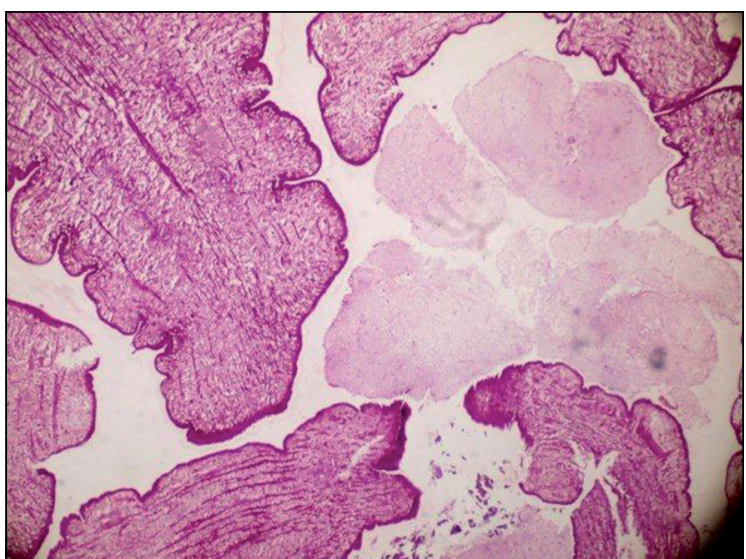


intermediarios secundarios crudos como culebras o ranas; contacto directo de carne cruda con heridas abiertas y mucosas e ingestión de larvas pleocercoides a través de los portadores ${ }^{2}$. De forma similar al reporte previo ${ }^{7}$ el paciente tenía antecedentes de ingestión frecuente de carne de serpiente, hospedero intermediario que en Cuba constituye en estos momentos la principal forma de infestación.

Aunque las manifestaciones clínicas son inespecíficas, las más frecuentes son las convulsiones de larga evolución. Puede existir historia de ingestión de hospederos intermediarios, crudos lo que ayuda al diagnóstico. Los síntomas de presentación del paciente fueron, por tanto, inusuales, lo cual depende de la localización pues la mayoría de estas lesiones se localizan en los hemisferios cerebrales (riesgo de convulsiones) y no en el tálamo ${ }^{2}$.

Los hallazgos imagenológicos tampoco fueron patognomónicos. Las características tomográficas de esta afección son: localización unilateral, zonas multifocales o extensivas de hipodensidad a lo largo de la sustancia blanca con atrofia cortical y dilatación ventricular homolateral, realce nodular o irregular con calcificaciones y migración de las lesiones en estudios seriados, usualmente vistas de 4 a 38 meses del estudio inicial. La resonancia magnética cerebral constituye el estudio imagenológico de elección por su capacidad de diagnóstico temprano y sus hallazgos incluyen degeneración y atrofia de la sustancia blanca y lesiones con intensidades mixtas. Se observa también el "signo del túnel" y lesiones en anillo multiloculadas. En nuestro paciente se observaron lesiones en anillo multiloculadas asociadas a edema (Figura 1 A y B). La localización talámica no fue encontrada en la mayoría de las series revisadas $1,2,5,6$.

El test de ELISA realizado para detectar la inmunoglobulina Sparganum-específica (Ig G) posee elevada sensibilidad. El estudio en el suero posee $88 \%$ de sensibilidad mientras que el del LCR, 93\%. En este paciente, dichos estudios fueron positivos (sustrato genérico para Spirometra spp.). Los resultados negativos del test de ELISA luego de la resección quirúrgica constituyen un elemento predictor de buen pronóstico como se observó en el paciente mientras que la persistencia de reacción positiva sugiere fuertemente la remoción incompleta del agente $^{8}$.

El tratamiento de elección consiste en la resección completa del verme. Esto es posible en caso de lesiones hemisféricas accesibles quirúrgicamente ${ }^{9}$. En cambio, la cirugía de lesiones en la región talámica tiene una elevada morbilidad. Durante el acto operatorio se aspiró un material alargado, elástico y blanquecino que levantó sospecha e incertidumbre en los cirujanos actuantes pero nunca se sospechó que se tratase de un verme y se interpretó como un fragmento de tejido capsular (Figura 2). Dicha muestra fue enviada al laboratorio de anatomía patológica y al ser procesada no se pudo conservar en su totalidad y no fue sometida a un análisis macroscópico parasitológico por un especialista en parasitología, con lo que quizás se hubiera evidenciado la posible presencia de escólex o proglótides que ayudaran a su identificación. La aspiración inesperada de este agente con la intención de realizar una biopsia pudo tener efecto terapéutico; por lo que debe tenerse en cuenta este recurso en caso de lesiones inaccesibles para su remoción total. Otros autores han reportado la aspiración estereotáctica como alternativa terapéutica ${ }^{9}$. Nosotros consideramos que la toma de muestras seriadas en diferentes direcciones y sobre todo en la región del nódulo realzado pudo ser el elemento clave para su logro.

Por otra parte, se ha planteado que el tratamiento sólo con prazicuantel no se asocia a buen pronóstico aunque se recomiendan altas dosis en pacientes inoperables 5 . Se requieren estudios imagenológicos secuenciales, tanto cuando se sospecha que el parásito es viable o no ${ }^{6}$.

\section{Conclusiones}

Aunque constituye una afección inusual, la esparganosis cerebral debe incluirse dentro del diagnóstico diferencial de lesiones múltiples con realce nodular.

Debe considerarse la aspiración estereotáctica no sólo como modalidad diagnóstica si no como terapéutica en localizaciones profundas e inaccesibles.

\section{Resumen}

Se presenta el caso clínico de un varón con 19 años de edad y el diagnóstico de una esparganosis cerebral. Consultó por cefalea y una hemiparesia izquierda. En una tomografía computarizada cerebral con contraste se observaron lesiones talámicas derechas. Se realizó una biopsia cerebral guiada por estereotaxia con aspiración completa de un verme. En el estudio histopatológico se planteó un probable parasitismo de SNC y fue tratado inicialmente con albendazol. Se confirmó la infección por Spirometra spp. por test de ELISA. Evolucionó con regresión de síntomas y remisión imagenológica de las lesiones. Este caso constituye el segundo reporte en Cuba de una infestación cerebral por este parásito y aspiración estereotáctica de la larva de Spirometra spp. 


\section{Referencias bibliográficas}

1.- Chen Y, Ye B. Sparganosis in China. Mehlhorn H, Wu Z, Ye B, editors. Treatment of Human Parasitosis in Traditional Chinese Medicine. Parasitology Research Monographs. Springer China 2014; 6: 169-83.

2.- Li M W, Song H Q, Li C, Lin H Y, Xie W T, Lin R Q, et al. Sparganosis in mainland China. Int J Infect Dis 2011; 15: 154-6.

3.- Duggal S, Mahajan RK, Duggal N, Hans C. Case of sparganosis: a diagnostic dilemma. Indian J Med Microbiol 2011; 29: 183-6.
4.- Chung S W, Kim Y H, Lee E J, Kim D H, Kim G Y. Two cases of pulmonary and pleural sparganosis confirmed by tissue biopsy and immunoserology. Braz J Infect Dis 2012; 16: $200-3$.

5.- Gonzenbach R R, Kong Y, Beck B, Buck A, Weller M, Semmler A. High-dose praziquantel therapy for cerebral sparganosis. J Neurol 2013; 260: 1423-5.

6.- Li Y X, Ramsahye H, Yin B, Zhang J, Geng D Y, Zee C S. Migration: a notable feature of cerebral sparganosis on follow-up MR imaging. Am J Neuroradiol 2013; 34: 327-33.

7.- Fernández Albán M, García Maeso I,
Figueredo Méndez J, Clará Morell G, Rodríguez Navas A, Mesa Santamarina A, et al. Resección estereotáctica de una larva viva de Sparganum mansonis en Cuba. Presentación de un caso. Rev Mex Neuroc 2009; 10: 4852.

8.- Cui J, Li N, Wang ZQ, Jianq P, Lin X M. Serodiagnosis of experimental sparganum infections of mice and human sparganosis by ELISA using ES antigens of Spirometra mansoni spargana. Parasitol Res 2011; 108: 1551-6.

9.- Deng L, Xiong P, Qian S. Diagnosis and stereotactic aspiration treatment of cerebral sparganosis: summary of 11 cases. J Neurosurg 2011; 114: 1421-5. 